\title{
Character String No Translations Data Type
}

National Cancer Institute

\section{Source}

National Cancer Institute. Character String No Translations Data Type. NCI Thesaurus.

Code C95631.

A data type comprised of a text string that can be displayed or machine processed, which does not allow translations. 